\title{
ON THE EXISTENCE OF PULLBACK ATTRACTOR FOR A TWO-DIMENSIONAL SHEAR FLOW WITH TRESCA'S BOUNDARY CONDITION
}

\author{
MAHDI BOUKROUCHE \\ Laboratory of Mathematics, University of Saint-Etienne, LaMUSE EA-3989 \\ 23 rue du Dr Paul Michelon, Saint-Etienne, 42023, France \\ E-mail: Mahdi.Boukrouche@univ-st-etienne.fr \\ GRZEGORZ ŁUKASZEWICZ \\ Mathematics Department, University of Warsaw \\ Banacha 2, 02-957 Warszawa, Poland \\ E-mail: glukasz@mimuw.edu.pl
}

\begin{abstract}
We consider a two-dimensional Navier-Stokes shear flow with time dependent boundary driving and subject to Tresca law. We establish the existence of a unique global in time solution and then, using a recent method based on the concept of the Kuratowski measure of noncompactness of a bounded set, we prove the existence of the pullback attractor for the associated cocycle. This research is motivated by a problem from lubrication theory.
\end{abstract}

1. Introduction. In this article we study the problem of existence of the pullback attractor for a class of two-dimensional turbulent boundary driven flows subject to the Tresca law which naturally appears in lubrication theory.

The problem is as follows. The flow of an incompressible fluid in a two-dimensional domain $\Omega$ is described by the equation of motion

$$
u_{t}-\nu \Delta u+(u \cdot \nabla) u+\nabla p=0 \quad \text { in } \quad \Omega
$$

and the incompressibility condition

$$
\operatorname{div} u=0 \quad \text { in } \Omega .
$$

2000 Mathematics Subject Classification: 76D05, 76F10, 76F20.

Key words and phrases: non-autonomous Navier-Stokes equations, lubrication theory, Tresca law, global in time solution, existence of pullback attractor.

This research was supported by Polish Government Grant MEiN 1 P303A 01730 and European Community Project FP6 EU SPADE2.

The paper is in final form and no version of it will be published elsewhere. 
To define the domain of the flow $\Omega$ consider first the channel

$$
\Omega_{\infty}=\left\{x=\left(x_{1}, x_{2}\right):-\infty<x_{1}<\infty, 0<x_{2}<h\left(x_{1}\right)\right\},
$$

where $h$ is a positive function, smooth, and L-periodic in $x_{1}$. Then

$$
\Omega=\left\{x=\left(x_{1}, x_{2}\right): 0<x_{1}<L, 0<x_{2}<h\left(x_{1}\right)\right\}
$$

and $\partial \Omega=\bar{\Gamma}_{0} \cup \bar{\Gamma}_{L} \cup \bar{\Gamma}_{1}$, where $\Gamma_{0}$ and $\Gamma_{1}$ are the bottom and the top, and $\Gamma_{L}$ is the lateral part of the boundary of $\Omega$.

We are interested in solutions of (1.1)-(1.2) in $\Omega$ which are L-periodic with respect to $x_{1}$. We assume that

$$
u=0 \quad \text { at } \Gamma_{1} .
$$

Moreover, we assume that there is no flux condition across $\Gamma_{0}$ so that the normal component of the velocity on $\Gamma_{0}$ satisfies

$$
u \cdot n=0 \text { at } \Gamma_{0},
$$

and that the tangential component of the velocity $u_{\eta}$ on $\Gamma_{0}$ is unknown and satisfies the Tresca law with a constant and positive friction coefficient $k$. This means that, c.f., e.g., [7],

$$
\left.\begin{array}{l}
\left|\sigma_{\eta}(u, p)\right|<k \Rightarrow u_{\eta}=U_{0}(t) e_{1} \\
\left|\sigma_{\eta}(u, p)\right|=k \Rightarrow \exists \lambda \geq 0 \text { such that } u_{\eta}=U_{0}(t) e_{1}-\lambda \sigma_{\eta}(u, p)
\end{array}\right\} \quad \text { at } \Gamma_{0}
$$

where $\sigma_{\eta}$ is the tangential component of the stress tensor on $\Gamma_{0}$ and

$$
t \mapsto U_{0}(t) e_{1}=\left(U_{0}(t), 0\right)
$$

is the time dependent velocity of the lower surface, producing the driving force of the flow. We suppose that $U_{0}$ is a locally Lipschitz continuous function of time $t$.

If $n=\left(n_{1}, n_{2}\right)$ is the unit outward normal to $\Gamma_{0}$, and $\eta=\left(\eta_{1}, \eta_{2}\right)$ is the unit tangent vector to $\Gamma_{0}$ then, using the summation convention, we have

$$
\sigma_{\eta}(u, p)=\sigma(u, p) \cdot n-((\sigma(u, p) \cdot n) \cdot n) n,
$$

where $\sigma_{i j}(u, p)=-p \delta_{i j}+\nu\left(u_{i, j}+u_{j, i}\right)$ is the stress tensor.

In the end, the initial condition for the velocity at time $\tau \in \mathbb{R}$ is

$$
u(x, \tau)=u_{0}(x) \text { for } \quad x \in \Omega .
$$

The problem is motivated by a flow in an infinite (rectified) journal bearing $\Omega \times$ $(-\infty,+\infty)$, where $\Gamma_{1} \times(-\infty,+\infty)$ represents the outer cylinder, and $\Gamma_{0} \times(-\infty,+\infty)$ represents the inner, rotating cylinder. In the lubrication problems the gap $h$ between cylinders is never constant. We can assume that the rectification does not change the equations as the gap between cylinders is very small with respect to their radii.

The system (1.1)-(1.2) with boundary conditions: (1.3) at $\Gamma_{1}$, for $h=$ const, and $u=$ const on $\Gamma_{0}$ instead of (1.4)-(1.5), was intensively studied in several contexts, some of them mentioned in the introduction of our paper [4]. The autonomous case with $h \neq$ const and with $u=$ const on $\Gamma_{0}$ was considered in [2], [3]. See also [4] where the case $h \neq$ const, $u=U(t) e_{1}$ on $\Gamma_{0}$, was considered. On the other hand, the important for applications 
dynamical problem we consider in this paper has not been studied earlier, neither in the autonomous nor in the nonautonomous case.

Our plan is as follows. First, we homogenize the boundary condition (1.5) by defining a smooth background flow, a simple version of the Hopf construction. In section 2 we study the variational formulation of the homogenized problem. In section 3 we prove existence and uniquness of a global in time solution of our problem. In section 4 we study the existence of the pullback attractor.

To homogenize the boundary condition (1.5) let

$$
u\left(x_{1}, x_{2}, t\right)=U\left(x_{2}, t\right) e_{1}+v\left(x_{1}, x_{2}, t\right)
$$

with

$$
U(0, t)=U_{0}(t), \quad U\left(h\left(x_{1}\right), t\right)=0, \quad x \in(0, L), \quad t \in(-\infty, \infty) .
$$

The new vector field $v$ is $L$-periodic in $x_{1}$ and satisfies the equation of motion

$$
v_{t}-\nu \Delta v+(v \cdot \nabla) v+\nabla p=G(v)
$$

with

$$
G(v)=-U v,_{x_{1}}-(v)_{2} U{, x_{2}} e_{1}+\nu U,_{x_{2} x_{2}} e_{1}-U,_{t} e_{1}
$$

where by $(v)_{2}$ we denoted the second component of $v$. As $\operatorname{div}\left(U e_{1}\right)=0$ we get

$$
\operatorname{div} v=0 \text { in } \Omega .
$$

From (1.8)-(1.9) we obtain

$$
v=0 \quad \text { on } \quad \Gamma_{1}
$$

and

$$
v \cdot n=0 \quad \text { on } \quad \Gamma_{0}
$$

Moreover, we have,

$$
\sigma_{\eta}(v, p)=\sigma_{\eta}(u, p)+\left(\left.\nu \frac{\partial U\left(x_{2}, t\right)}{\partial x_{2}}\right|_{x_{2}=0}, 0\right) .
$$

Since we can define the extension $U$ in such a way that

$$
\left.\frac{\partial U\left(x_{2}, t\right)}{\partial x_{2}}\right|_{x_{2}=0}=0
$$

the Tresca condition (1.5) transforms to

$$
\left.\begin{array}{l}
\left|\sigma_{\eta}(v, p)\right|<k \Rightarrow v_{\eta}=0 \\
\left|\sigma_{\eta}(v, p)\right|=k \Rightarrow \exists \lambda \geq 0 \text { such that } v_{\eta}=-\lambda \sigma_{\eta}(v, p)
\end{array}\right\} \text { at } \Gamma_{0}
$$

In the end the initial condition becomes

$$
v(x, \tau)=v_{0}(x)=u_{0}(x)-U\left(x_{2}, \tau\right) e_{1} .
$$

2. Variational formulation of the problem. In this section we present the variational formulation of the homogenized problem (1.10)-(1.15). Then, for the convenience of the readers, we describe the relations between the classical and the weak formulations.

We begin with some basic definitions of the paper. 
Let

$$
\begin{gathered}
\tilde{V}=\left\{v \in \mathcal{C}^{\infty}(\Omega)^{2}: \operatorname{div} v=0 \text { in } \Omega, v \text { is L-periodic in } x_{1},\right. \\
\left.v=0 \text { at } \Gamma_{1}, \quad v \cdot n=0 \text { at } \Gamma_{0}\right\}
\end{gathered}
$$

and

$$
V=\text { closure of } \tilde{V} \text { in } H^{1}(\Omega)^{2}, \quad H=\text { closure of } \tilde{V} \text { in } L^{2}(\Omega)^{2} \text {. }
$$

We define scalar products in $H$ and $V$, respectively, by

$$
(u, v)=\int_{\Omega} u(x) v(x) d x \quad \text { and } \quad(\nabla u, \nabla v)
$$

and their associated norms by

$$
|v|=(v, v)^{\frac{1}{2}} \quad \text { and } \quad\|v\|=(\nabla v, \nabla v)^{\frac{1}{2}} .
$$

Let, for $u, v$ and $w$ in $V$

$$
a(u, v)=(\nabla u, \nabla v) \quad \text { and } \quad b(u, v, w)=((u \cdot \nabla) v, w) .
$$

In the end, let us define the functional $j$ on $V$ by

$$
j(u)=\int_{\Gamma_{0}} k\left|u\left(x_{1}, 0\right)\right| d x_{1} .
$$

The variational formulation of the homogenized problem (1.10)-(1.15) is as follows.

Problem 2.1. Given $\tau \in \mathbb{R}$ and $v_{0} \in H$, find $v:(\tau, \infty) \rightarrow H$ such that:

(i) for all $T>\tau$,

$$
v \in \mathcal{C}([\tau, T] ; H) \cap L^{2}(\tau, T ; V), \quad \text { with } \quad v_{t} \in L^{2}\left(\tau, T ; V^{\prime}\right)
$$

(ii) for all $\Theta$ in $V$, all $T>\tau$, and for almost all $t$ in the interval $[\tau, T]$, the following variational inequality holds

$$
\begin{array}{r}
\left\langle v_{t}(t), \Theta-v(t)\right\rangle+\nu a(v(t), \Theta-v(t))+b(v(t), v(t), \Theta-v(t)) \\
+j(\Theta)-j(v(t)) \geq(\mathcal{L}(v(t)), \Theta-v(t))
\end{array}
$$

(iii) the initial condition

$$
v(x, \tau)=v_{0}(x) .
$$

holds.

In (2.1) the functional $\mathcal{L}(v(t))$ is defined for almost all $t \geq \tau$ by,

$$
\begin{aligned}
(\mathcal{L}(v(t)), \Theta)= & -\nu a(\xi, \Theta)-\left(\xi_{, t}(t), \Theta\right) \\
& -b(\xi(t), v(t), \Theta)-b(v(t), \xi(t), \Theta),
\end{aligned}
$$

where $\xi=U e_{1}$ is a suitable smooth background flow.

We have the following relations between classical and weak formulations.

Proposition 2.1. Every classical solution of problem (1.10)-(1.15) is also a solution of Problem 2.1. On the other hand, every solution of Problem 2.1 which is smooth enough is also a classical solution of problem (1.10)-(1.15). 
Proof. Let $v$ be a classical solution of problem (1.10)-(1.15). As it is (by assumption) sufficiently regular, we have to check only (2.1). Remark first that (1.10) can be written as

$$
v_{t}-\sigma_{i j, j}(v, p)+(v \cdot \nabla) v=G(v(t)) .
$$

Let $\Theta \in V$. Mutiplying (2.4) by $\Theta-v(t)$ and using Green's formula we obtain

$$
\begin{array}{r}
\left\langle v_{t}, \Theta-v(t)\right\rangle+\int_{\Omega} \sigma_{i j}(v, p)(\Theta-v(t))_{i, j} d x+b(v(t), v(t), \Theta-v(t)) \\
=\int_{\partial \Omega} \sigma_{i j}(v, p) n_{j}(\Theta-v(t))_{i}+\langle G(v(t)), \Theta-v(t)\rangle \text { for almost all } t \in[\tau, T] .
\end{array}
$$

As $v(t)$ and $\Theta$ are in $V$, we have

$$
\int_{\Omega} \sigma_{i j}(v, p)(\Theta-v(t))_{i, j} d x=\nu a(v(t), \Theta-v(t))
$$

and using (1.6) we obtain

$$
\int_{\partial \Omega} \sigma_{i j}(v, p) n_{j}(\Theta-v(t))_{i}=\int_{\Gamma_{0}} \sigma_{\eta}(v, p) \cdot\left(\Theta-v_{\eta}(t)\right)-\int_{\Gamma_{0}}\left(\sigma_{i j} n_{j} n_{i}\right) n_{i}\left(\Theta-v_{\eta}(t)\right)_{i}
$$

As $n_{i}\left(\Theta-v_{\eta}(t)\right)_{i}=0$ on $\Gamma_{0}$, we get

$$
\begin{aligned}
\int_{\partial \Omega} \sigma_{i j}(v, p) n_{j}(\Theta-v(t))_{i}= & \int_{\Gamma_{0}} \sigma_{\eta}(v, p) \cdot\left(\Theta-v_{\eta}(t)\right)=\int_{\Gamma_{0}}\left(\sigma_{\eta} \cdot \Theta+k|\Theta|\right) \\
& -\int_{\Gamma_{0}} k\left(|\Theta|-\left|v_{\eta}(t)\right|\right)-\int_{\Gamma_{0}}\left(k\left|v_{\eta}(t)\right|+\sigma_{\eta} \cdot v_{\eta}(t)\right) .
\end{aligned}
$$

Remark that $\sigma_{\eta} \cdot \Theta+k|\Theta| \geq 0$, and the Tresca condition (1.14) is equivalent [7] to

$$
k\left|v_{\eta}(t)\right|+\sigma_{\eta} \cdot v_{\eta}(t)=0 \quad \text { a.e } \quad \text { on } \quad \Gamma_{0} .
$$

Thus

$$
\int_{\partial \Omega} \sigma_{i j}(v, p) n_{j}(\Theta-v(t))_{i} \geq-\int_{\Gamma_{0}} k\left(|\Theta|-\left|v_{\eta}(t)\right|\right)=-j(\Theta)+j(v(t)) .
$$

As

$$
\langle G(v(t)), \Theta-v(t)\rangle=(\mathcal{L}(v(t)), \Theta-v(t))
$$

from (2.6) and (2.7) we see that (2.5) becomes (2.1), and (2.2) is the same as (1.15).

Conversely, suppose that $v$ is a solution to Problem 2.1 and let $\varphi$ be in the space $\left(H_{\text {div }}^{1}(\Omega)\right)^{2}=\{\varphi \in V: \varphi=0$ on $\Gamma\}$. We take $\Theta=v(t) \pm \varphi$ in (2.1), and using the Green formula, we obtain

$$
\left\langle v_{t}-\nu \Delta v(t)+v(t) \cdot \nabla v(t)-G(v(t)), \varphi\right\rangle=0 \quad \forall \varphi \in\left(H_{d i v}^{1}(\Omega)\right)^{2} .
$$

Thus, there exists $p \in H^{-1}(\Omega)$ such that

$$
v_{t}-\nu \Delta v(t)+v(t) \cdot \nabla v(t)-G(v(t))=\nabla p \quad \text { a.e. in } \Omega .
$$

so that (1.10) holds. We obtain (1.14) as in [1], and we have immediately (1.11)-(1.13) and (1.15). 
3. Existence and uniqueness of a global in time solution. In this section we establish the existence and uniqueness of a global in time solution for Problem 2.1. First, we present three lemmas.

Lemma $3.1([4])$. For any $t \in \mathbb{R}$ consider the smooth extension

$$
\xi\left(x_{2}, t\right)=U\left(x_{2}, t\right) e_{1}=U_{0}(t) \rho\left(x_{2} /\left(h_{0} \varepsilon(t)\right)\right) e_{1} .
$$

of $U_{0}(t) e_{1}$ from $\Gamma_{0}$ to $\Omega$, where

$$
\varepsilon=\varepsilon(t)= \begin{cases}2 & \text { if }\left|U_{0}(t)\right| \leq \nu /\left(8 h_{0}\right) \\ \nu /\left(4 h_{0}\left|U_{0}(t)\right|\right) & \text { if }\left|U_{0}(t)\right| \geq \nu /\left(8 h_{0}\right)\end{cases}
$$

$h_{0}=\min _{0 \leq x_{1} \leq L} h\left(x_{1}\right)$, and $\rho:[0, \infty) \rightarrow[0,1]$ is a smooth function such that

$$
\rho(0)=1, \quad \rho^{\prime}(0)=0, \quad \operatorname{supp} \rho \subset[0,1 / 2], \quad \max \left|\rho^{\prime}(s)\right| \leq \sqrt{8} .
$$

Then we have

$$
|b(v, \xi(t), v)| \leq \frac{\nu}{4}\|v\|^{2} \quad \text { for all }, v \in V
$$

and

$$
\left.\frac{\partial U\left(x_{2}, t\right)}{\partial x_{2}}\right|_{x_{2}=0}=0 .
$$

Lemma 3.2 ([4]). Let $U$ be as in Lemma 3.1. Then for almost all $t$,

$$
\begin{aligned}
|\xi(t)|^{2} & =\int_{\Omega}\left|U\left(x_{2}, t\right)\right|^{2} d x_{1} d x_{2} \leq \frac{1}{2} L h_{0} U_{0}^{2}(t) \varepsilon(t), \\
|\nabla \xi(t)|^{2} & =\int_{\Omega}\left|U_{x_{2}}\left(x_{2}, t\right)\right|^{2} d x_{1} d x_{2} \leq \frac{4 L U_{0}^{2}(t)}{h_{0}} \frac{1}{\varepsilon(t)},
\end{aligned}
$$

and

$$
|\xi, t|^{2}=\int_{\Omega}\left|U_{t}\left(x_{2}, t\right)\right|^{2} d x_{1} d x_{2} \leq\left(\left|U_{0}^{\prime}(t)\right|+\sqrt{2}\left|U_{0}(t)\right| \frac{\left|\varepsilon^{\prime}(t)\right|}{\varepsilon(t)}\right)^{2} \frac{L h_{0} \varepsilon(t)}{2} .
$$

LEMMA 3.3. For all $v$ in $V$ we have the anisotropic Ladyzhenskaya inequality

$$
\|v\|_{L^{4}(\Omega)} \leq C(\Omega)|v|^{\frac{1}{2}}\|v\|^{\frac{1}{2}} .
$$

Proof. Let $v \in V$ and $\xi \in C^{1}(]-L, L[)$ such that $\xi=1$ on $[0, L]$ and $\xi=0$ at $x_{1}=-L$. Define $\varphi=\xi v$, and extend $\varphi$ by 0 to $\left.\Omega_{1}=\right]-L, L[\times] 0, h\left[\right.$, where $h=\max _{0 \leq x_{1} \leq L} h\left(x_{1}\right)$. We obtain

$$
\varphi^{2}\left(x_{1}, x_{2}\right)=2 \int_{-L}^{x_{1}} \varphi\left(t_{1}, x_{2}\right) \frac{\partial \varphi}{\partial t_{1}}\left(t_{1}, x_{2}\right) d t_{1} \leq 2 \int_{-L}^{L}\left|\varphi\left(x_{1}, x_{2}\right)\right|\left|\frac{\partial \varphi}{\partial x_{1}}\left(x_{1}, x_{2}\right)\right| d x_{1}
$$

and

$$
\varphi^{2}\left(x_{1}, x_{2}\right)=-2 \int_{x_{2}}^{h} \varphi\left(x_{1}, t_{2}\right) \frac{\partial \varphi}{\partial t_{2}}\left(x_{1}, t_{2}\right) d t_{2} \leq 2 \int_{0}^{h}\left|\varphi\left(x_{1}, x_{2}\right)\right|\left|\frac{\partial \varphi}{\partial x_{2}}\left(x_{1}, x_{2}\right)\right| d x_{2},
$$


whence

$$
\begin{aligned}
\|\varphi\|_{L^{4}\left(\Omega_{1}\right)}^{4} & =\int_{\Omega_{1}} \varphi^{2}\left(x_{1}, x_{2}\right) \varphi^{2}\left(x_{1}, x_{2}\right) d x_{1} d x_{2} \\
& \leq\left(\int_{0}^{h} \sup _{-L \leq x_{1} \leq L} \varphi^{2}\left(x_{1}, x_{2}\right) d x_{2}\right)\left(\int_{-L}^{L} \sup _{0 \leq x_{2} \leq h} \varphi^{2}\left(x_{1}, x_{2}\right) d x_{1}\right) \\
& \leq 4\left(\int_{0}^{h} \int_{-L}^{L}|\varphi|\left|\frac{\partial \varphi}{\partial x_{1}}\right| d x_{1} d x_{2}\right) \times\left(\int_{0}^{h} \int_{-L}^{L}|\varphi|\left|\frac{\partial \varphi}{\partial x_{2}}\right| d x_{2} d x_{1}\right)
\end{aligned}
$$

By the Cauchy-Schwarz inequality,

$$
\begin{aligned}
\|\varphi\|_{L^{4}\left(\Omega_{1}\right)}^{4} & \leq 4|\varphi|_{L^{2}\left(\Omega_{1}\right)}^{2}\left|\frac{\partial \varphi}{\partial x_{1}}\right|_{L^{2}\left(\Omega_{1}\right)}\left|\frac{\partial \varphi}{\partial x_{2}}\right|_{L^{2}\left(\Omega_{1}\right)} \\
& \leq 2|\varphi|_{L^{2}\left(\Omega_{1}\right)}^{2}\left(\left|\frac{\partial \varphi}{\partial x_{1}}\right|_{L^{2}\left(\Omega_{1}\right)}^{2}+\left|\frac{\partial \varphi}{\partial x_{2}}\right|_{L^{2}\left(\Omega_{1}\right)}^{2}\right) \\
& \leq 2|\varphi|_{L^{2}\left(\Omega_{1}\right)}^{2}|\nabla \varphi|_{L^{2}\left(\Omega_{1}\right)}^{2}
\end{aligned}
$$

We use $|\xi| \leq 1$ and the Poincaré inequality to get

$$
\|v\|_{L^{4}(\Omega)} \leq\|\varphi\|_{L^{4}\left(\Omega_{1}\right)}, \quad|\varphi|_{L^{2}\left(\Omega_{1}\right)} \leq 2|v|_{L^{2}(\Omega)} \quad \text { and } \quad|\nabla \varphi|_{L^{2}\left(\Omega_{1}\right)} \leq C\|v\|_{V}
$$

for some constant $C$, whence (3.1) holds.

THEOREM 3.1. Let $v_{0} \in H$ and the function $s \mapsto\left|U_{0}(s)\right|^{3}+\left|U_{0}^{\prime}(s)\right|^{2}$ be locally integrable on the real line. Then there exists a solution of Problem 2.1.

Proof. We provide only the main steps of the proof as it is quite standard and, on the other hand, long. The estimates we obtain will be used further in the paper.

Observe that the functional $j$ is convex but nondifferentiable. To overcome this difficulty we use the following approach (see, i.e., [8]). For $\delta>0$ let $j_{\delta}: V \rightarrow \mathbb{R}$ be a functional defined by

$$
\varphi \mapsto j_{\delta}(\varphi)=\frac{1}{1+\delta} \int_{\Gamma_{0}} k|\varphi|^{1+\delta} d x
$$

which is convex, lower continuous and finite on $V$, and has the following properties

- $\exists \chi \in V^{\prime}$ and $\mu \in \mathbb{R}$ such that $j_{\delta}(\varphi) \geq\langle\chi, \varphi\rangle+\mu \quad \forall \varphi \in V$,

- $\lim _{\delta \rightarrow 0^{+}} j_{\delta}(\varphi)=j(\varphi) \quad \forall \varphi \in V$,

- $v_{\delta} \rightarrow v$ (weakly) in $V \Rightarrow \lim _{\delta \rightarrow 0^{+}} j_{\delta}\left(v_{\delta}\right) \geq j(v)$.

The functional $j_{\delta}$ is Gâteaux differentiable in $V$, with

$$
\left(j_{\delta}^{\prime}(v), \Theta\right)=\int_{\Gamma_{0}} k|v|^{\delta-1} v \Theta d x \quad \forall \Theta \in V .
$$

Let us consider the following equation

$$
\begin{aligned}
\left(\frac{d v_{\delta}}{d t}, \Theta\right) & +\nu a\left(v_{\delta}(t), \Theta\right)+b\left(v_{\delta}(t), v_{\delta}(t), \Theta\right)+\left(j_{\delta}^{\prime}\left(v_{\delta}\right), \Theta\right) \\
& =-\nu a(\xi(t), \Theta)-(\xi, t, \Theta)-b\left(\xi(t), v_{\delta}(t), \Theta\right)-b\left(v_{\delta}(t), \xi(t), \Theta\right)
\end{aligned}
$$

with initial condition

$$
v_{\delta}(\tau)=v_{0}
$$


For $\delta>0$, we establish an a priori estimate of $v_{\delta}$. Since $\left(j_{\delta}^{\prime}\left(v_{\delta}\right), v_{\delta}\right) \geq 0, v_{\delta}(t) \in V$, and $b\left(v_{\delta}(t), v_{\delta}(t), v_{\delta}(t)\right)=b\left(\xi, v_{\delta}(t), v_{\delta}(t)\right)=0$ then taking $\Theta=v_{\delta}(t)$ in (3.2) we get

$$
\begin{aligned}
\frac{1}{2} \frac{d}{d t}\left|v_{\delta}(t)\right|^{2}+\nu\left\|v_{\delta}(t)\right\|^{2} \leq & \left(-\nu a\left(\xi(t), v_{\delta}(t)\right)\right. \\
& -\left(\xi_{, t}, v_{\delta}(t)\right)-b\left(v_{\delta}(t), \xi(t), v_{\delta}(t)\right)
\end{aligned}
$$

In view of estimate (3.1) and the Poincaré inequality we obtain from (3.4)

$$
\frac{1}{2} \frac{d}{d t}\left|v_{\delta}(t)\right|^{2}+\frac{\nu}{2}\left\|v_{\delta}(t)\right\|^{2} \leq 2 \nu\|\xi\|^{2}+\frac{1}{\nu \lambda_{1}}|\xi, t|^{2} .
$$

where $\lambda_{1}$ is the first eigenvalue of the Stokes operator in our problem. We estimate the right hand side in terms of the data using Lemma 3.2 with $\varepsilon$ as in Lemma 3.1 to get

$$
\frac{1}{2} \frac{d}{d t}\left|v_{\delta}(t)\right|^{2}+\frac{\nu}{2}\left\|v_{\delta}(t)\right\|^{2} \leq F(t)
$$

with

$$
\begin{aligned}
F(t)= & 2 \nu\left(\frac{1}{2} L h_{0} U_{0}^{2}(t) \varepsilon(t)+\frac{4 L U_{0}^{2}(t)}{h_{0} \varepsilon(t)}\right) \\
& +\frac{1}{\nu \lambda_{1}}\left(\left|U_{0}^{\prime}(t)\right|+\sqrt{2}\left|U_{0}(t)\right| \frac{\left|\varepsilon^{\prime}(t)\right|}{\varepsilon(t)}\right)^{2} \frac{L h_{0} \varepsilon(t)}{2} \\
= & c_{1}(\nu, \Omega)\left(\left|U_{0}(t)\right|^{3}+\left|U_{0}^{\prime}(t)\right|^{2}\right) .
\end{aligned}
$$

From (3.5) we conclude that

$$
\left|v_{\delta}(t)\right|^{2}+\nu \int_{\tau}^{t}\left\|v_{\delta}(s)\right\|^{2} d s \leq|v(\tau)|^{2}+2 \int_{\tau}^{t} F(s) d s .
$$

As the function $F$ is, by assumption, locally integrable on the real line, we deduce that

$$
v_{\delta} \text { is bounded in } L^{2}(\tau, T ; V) \cap L^{\infty}(\tau, T ; H) \text {, independently of } \delta \text {. }
$$

The existence of $v_{\delta}$ satisfying (3.2)-(3.3) is based on inequality (3.5), the Galerkin approximations, and the compactness method. Moreover from (3.7) we can deduce that

$$
\frac{d v_{\delta}}{d t} \quad \text { is bounded in } L^{2}\left(\tau, T ; V^{\prime}\right) \text {. }
$$

From (3.8) and from (3.9) we conclude that there exists $v$ such that (possibly for a subsequence)

$$
v_{\delta} \rightarrow v \quad \text { in } \quad L^{2}(\tau, T ; V), \quad \text { and } \quad \frac{d v_{\delta}}{d t} \rightarrow \frac{d v}{d t} \quad \text { in } L^{2}\left(\tau, T ; V^{\prime}\right) \text { (weakly) }
$$

In view of $(3.10), v \in \mathcal{C}([\tau, T] ; H)$, and

$$
v_{\delta} \rightarrow v \quad \text { in } \quad L^{2}(\tau, T ; H) \quad \text { strongly. }
$$

We can now pass to the limit $\delta \rightarrow 0$ in (3.2)-(3.3) exactly as in [7] to obtain the variational inequality (2.1) for almost every point $t \in] \tau, T$ [. Thus the existence of a solution of Problem 2.1 is established.

Theorem 3.2. Under the hypotheses of Theorem 3.1, the solution $v$ of Problem 2.1 is unique and the map $v(\tau) \rightarrow v(t)$, for $t>\tau$, is continuous in $H$. 
Proof. Let $v$ and $w$ be two solutions of Problem 2.1. Then for $u(t)=w(t)-v(t)$ we have

$$
\frac{1}{2} \frac{d}{d t}|u(t)|^{2}+\nu\|u(t)\|^{2} \leq b(u(t), w(t), u(t))+b(u(t), \xi(t), u(t))
$$

By Lemma 3.1 and the anisotropic Ladyzhenskaya inequality (3.1) we obtain

$$
\frac{d}{d t}|u(t)|^{2}+\frac{\nu}{4}\|u(t)\|^{2} \leq \frac{4}{\nu} C(\Omega)\|w(t)\|^{2}|u(t)|^{2},
$$

and in view of the Poincaré inequality we conclude

$$
\frac{d}{d t}|u(t)|^{2}+\frac{\sigma}{4}|u(t)|^{2} \leq \frac{4}{\nu} C(\Omega)\|w(t)\|^{2}|u(t)|^{2} .
$$

Using again the Gronwall lemma, we obtain

$$
|u(t)|^{2} \leq[u(\tau)]^{2} \exp \left\{-\int_{\tau}^{t}\left(\frac{\sigma}{4}-\frac{4}{\nu} C(\Omega)\|w(s)\|^{2}\right) d s\right\} .
$$

From (3.10) it follows that the solution $w$ of Problem 2.1 belongs to $L^{2}(\tau, t ; V)$. We have thus proved that the map $v(\tau) \rightarrow v(t), t>\tau$, in $H$ is continuous. In particular, as $u(\tau)=w(\tau)-v(\tau)=0$, the solution $v$ of Problem 2.1 is unique. This ends the proof of Theorem 3.2.

4. Existence of the pullback attractor. In this section we prove existence of the pullback attractor for the cocycle associated to the considered problem by using a method based on the concept of the Kuratowski measure of noncompactness of a bounded set. This method is very useful when one deals with variational inequalities as it overcomes obstacles coming from the usual methods. One needs neither compactness of the dynamics which results from the second energy inequality nor asymptotic compactness, cf., i.e., [4, $5,6,11]$, which results from the energy equation. In the case of variational inequalities it is much more difficult to obtain the second energy inequality due to presence of boundary functionals, on the other hand, we do not have any energy equation.

First we recall the definition of a cocycle and a pullback attractor, then, following [10], recall the abstract theory, and in the end apply the latter to our problem.

Definition 4.1. Let $\mathcal{P}$ be a metric space and $\theta: \mathbb{R} \times \mathcal{P} \rightarrow \mathcal{P}$ be a mapping such that $\theta_{t}: \mathcal{P} \rightarrow \mathcal{P}$ form a group, namely,

$$
\theta_{0}=I d, \quad \text { and } \quad \theta_{t+r}=\theta_{t} \circ \theta_{r} \quad \forall t, r \in \mathbb{R} .
$$

Let $H$ be a complete metric space. A mapping $\Phi: \mathbb{R}_{+} \times \mathcal{P} \times H \rightarrow H$ is said to be a cocycle on $H$ with respect to a group $\theta$ if

$$
\begin{gathered}
\Phi\left(0, p, v_{0}\right)=v_{0} \quad \forall v_{0} \in H, \quad p \in \mathcal{P}, \\
\Phi\left(t+r, p, v_{0}\right)=\Phi\left(t, \theta_{r}(p), \Phi\left(r, p, v_{0}\right)\right), \quad \forall t, r \in \mathbb{R}_{+}, \quad p \in \mathcal{P} .
\end{gathered}
$$

Definition 4.2. A family $\mathcal{A}=\left\{\mathcal{A}_{p}\right\}_{p \in \mathcal{P}}$ of nonempty compact sets of $H$ is called a pullback attractor of the $\theta$-cocycle $\Phi$ if it is $\Phi$-invariant, that is,

$$
\Phi\left(t, p, \mathcal{A}_{p}\right)=\mathcal{A}_{\theta_{t}(p)} \quad \forall(t, p) \in \mathbb{R}_{+} \times \mathcal{P},
$$

and pullback attracting, that is,

$$
\lim _{t \rightarrow \infty} \operatorname{dist}\left(\Phi\left(t, \theta_{-t}(p), B\right), \mathcal{A}_{p}\right)=0 \quad \forall B \in B(H), \quad \forall p \in \mathcal{P} .
$$


where $B(H)$ is the set of all bounded subsets of $H$, and dist is the Hausdorff semidistance between $X$ and $Y$ defined by

$$
\operatorname{dist}(X, Y)=\sup _{x \in X} \inf _{y \in Y} d(x, y) .
$$

Definition 4.3. The pullback $\omega$-limit set $\omega_{p}(B)$ of $B$ is defined, for any $t \geq 0$, by the following

$$
\omega_{p}(B)=\bigcap_{s \geq 0} \overline{\bigcup_{t \geq s} \Phi\left(t, \theta_{-t}(p), B\right)} .
$$

The other definitions and theorems we need are as follows. Let $B \in B(H)$. The Kuratowski measure of noncompactness [9] is defined by

$$
\alpha(B)=\inf \{\delta: B \text { admits a finite cover by sets of diameter } \leq \delta\} .
$$

Definition 4.4 ([10]). A cocycle $\Phi$ on $H$ is said to be pullback $\omega$-limit compact if for any $B \in B(H)$, for any $p \in \mathcal{P}$,

$$
\lim _{\tau \rightarrow \infty} \alpha\left(\bigcup_{t \geq \tau} \Phi\left(t, \theta_{-t}(p), B\right)\right)=0 .
$$

Definition 4.5 ([10]). Let $H$ be a Banach space. A cocycle $\Phi$ is said to be norm-to-weak continuous on $H$ if for all $(t, p, x) \in \mathbb{R}_{+} \times \mathcal{P} \times H$ and for every sequence $\left(x_{n}\right) \in H$,

$$
x_{n} \rightarrow x \quad \text { strongly in } H \Rightarrow \Phi\left(t, p, x_{n}\right) \rightarrow \Phi(t, p, x) \quad \text { weakly in } H .
$$

Theorem 4.1 ([10]). Let $H$ be a Banach space. Let $\Phi$ be a cocycle on $H$ with respect to a group $\theta$. If $\Phi$ is norm-to-weak continuous and possesses a uniformly absorbing set $B_{0}$, then $\Phi$ possesses a pullback attractor $\mathcal{A}=\left\{\mathcal{A}_{p}\right\}_{p \in \mathcal{P}}$, satisfying

$$
\mathcal{A}_{p}=\omega_{p}\left(B_{0}\right), \quad \forall p \in \mathcal{P},
$$

if and only if it is pullback $\omega$-limit compact.

THEOREM 4.2 ([10]). Let $H$ be a Banach space. If the cocycle $\Phi$ satisfies the pullback condition (PC): for any $p \in \mathcal{P}, B \in B(H)$ and $\varepsilon>0$, there exists $t_{0}(p, B, \varepsilon)$ and a finite dimensional subspace $E$ of $H$ such that for a bounded projector $P: H \rightarrow E$,

$$
\begin{gathered}
P\left(\bigcup_{t \geq t_{0}} \Phi\left(t, \theta_{-t}(p), B\right)\right) \quad \text { is bounded, } \quad \forall t \in \mathbb{R}_{+}, \\
\left|(I-P)\left(\bigcup_{t \geq t_{0}} \Phi\left(t, \theta_{-t}(p), x\right)\right)\right| \leq \varepsilon, \quad \forall x \in B,
\end{gathered}
$$

then $\Phi$ is pullback $\omega$-limit compact.

Now, we define a cocycle $\Phi: \mathbb{R}_{+} \times \mathbb{R} \times H \rightarrow H$ as the evolutionary process $\mathbf{U}$ associated with Problem 2.1, namely,

$$
\Phi\left(s, t, v_{0}\right)=\mathbf{U}(s+t, t) v_{0}
$$

with $\theta_{s}(t)=t+s$. In particular,

$$
\Phi\left(s, \theta_{-s}(t), v_{0}\right)=\mathbf{U}\left(\theta_{-s}(t)+s, \theta_{-s}(t)\right) v_{0}=\mathbf{U}(t, t-s) v_{0} .
$$


LEMMA 4.1. Let

$$
\sup _{h \in \mathbb{R}} \int_{h}^{h+1} F(s) d s<R(F)<\infty,
$$

$\sigma>0$ and $t \geq \tau$. Then for every $\varepsilon>0$ there exists $\delta=\delta(\varepsilon)>0$ such that

$$
\int_{\tau}^{t} e^{-\sigma(t-s)} F(s) d s \leq \frac{\varepsilon}{2}+\frac{e^{-\sigma \delta}}{1-e^{-\sigma}} R(F) .
$$

Proof. Let $\delta$ be such that $\int_{t-\delta}^{t} F(s) d s \leq \frac{\varepsilon}{2}, \tau<\delta<t$. Then, by (4.1),

$$
\int_{\tau}^{t} e^{-\sigma(t-s)} F(s) d s \leq \frac{\varepsilon}{2}+\sum_{k=1}^{\infty} \int_{t-(\delta+k+1)}^{t-(\delta+k)} e^{-\sigma(t-s)} F(s) d s \leq \frac{\varepsilon}{2}+\frac{e^{-\sigma \delta}}{1-e^{-\sigma}} R(F) .
$$

Let $F(s)=\left|U_{0}(s)\right|^{3}+\left|U_{0}^{\prime}(s)\right|^{2}$ be as in (3.6). Then we have

Lemma 4.2. Let the initial condition $v_{0}$ in Problem 2.1 belong to a ball $B(0, \rho)$ in $H$. Suppose that (4.1) holds. Then the solution $v$ of Problem 2.1 satisfies

$$
\sup _{h \geq \tau} \int_{h}^{h+1}\|v(s)\|^{2} d s \leq \frac{2}{\nu}\left\{\rho^{2}+\left(1+\frac{e^{-\sigma \delta}}{1-e^{-\sigma}}\right) R(F)\right\} .
$$

Proof. Taking $\Theta=0$ in (2.1) we obtain, similarly as in the proof of Theorem 3.1,

$$
\frac{1}{2} \frac{d}{d t}|v(t)|^{2}+\frac{\nu}{2}\|v(t)\|^{2} \leq F(t)
$$

and, in consequence,

$$
\frac{1}{2} \frac{d}{d t}|v(t)|^{2}+\frac{\sigma}{2}|v(t)|^{2} \leq F(t)
$$

with $\sigma=\nu \lambda_{1}$. By Gronwall's inequality and Lemma 4.1 with $\varepsilon=\rho^{2}$ we conclude from the last inequality that for $t \geq \tau$,

$$
|v(t)|^{2} \leq 2 \rho^{2}+\frac{2 e^{-\sigma \delta}}{1-e^{-\sigma}} R(F)
$$

By integration of (4.4) we obtain the first energy estimate: for $\tau \leq \eta \leq t$,

$$
|v(t)|^{2}+\nu \int_{\eta}^{t}\|v(s)\|^{2} d s \leq 2 \int_{\eta}^{t} F(s) d s+|v(\eta)|^{2} .
$$

Using this estimate and (4.6) we obtain (4.3).

THEOREM 4.3. Let $v_{0} \in H$ and $U_{0}$ be such that (4.1) holds, with $F(s)=\left|U_{0}(s)\right|^{3}+$ $\left|U_{0}^{\prime}(s)\right|^{2}$. Then there exists a pullback attractor $\mathcal{A}$ in the sense of Theorem 4.1 for the cocycle $\Phi$ given by the evolutionary process $\mathbf{U}(t, \tau)$ above.

Proof. From (4.5), (4.6), the Gronwall inequality, and Lemma 4.1 we obtain

$$
\left|\mathbf{U}(s+t, t) v_{0}\right|^{2} \leq e^{-\sigma s}\left|v_{0}\right|^{2}+\varepsilon+\frac{2 e^{-\sigma \delta}}{1-e^{-\sigma}} R(F) .
$$

For $v_{0}$ in $B(0, \rho)$ and $s$ large enough, $\mathbf{U}(s+t, t) v_{0} \in B\left(0, \rho_{0}\right)$, where $\rho_{0}$ depends only on $\varepsilon, \rho$, and $R(F)$, which means that there exists a uniformly absorbing ball in $H$.

From (3.11) it follows that the evolutionary process $\mathbf{U}$ is strongly continuous in $H$, whence, in particular, it is norm-to-weak continuous on $H$. 
Thus, according to Theorem 4.1 and Theorem 4.2, to finish the proof we have to prove that the pullback condition (PC) holds.

Let $A$ be the Stokes operator in $H$. As $A^{-1}$ is continuous and compact in $H$, there exists a sequence $\left\{\lambda_{j}\right\}_{j=1}^{\infty}$ such that

$$
0<\lambda_{1} \leq \lambda_{2} \leq \cdots \leq \lambda_{j} \leq \ldots \quad \text { with } \lim _{j \rightarrow+\infty} \lambda_{j}=\infty
$$

and a family of elements $\left\{\varphi_{j}\right\}_{j=1}^{\infty}$ of $D(A)$, which are orthonormal in $H$ such that $A \varphi_{j}=$ $\lambda_{j} \varphi_{j}$

We define the m-dimensional subspace $V_{m}$, of $V$, and the orthogonal projection operator $P_{m}: V \rightarrow V_{m}$ by

$$
V_{m}=\operatorname{span}\left\{\varphi_{1}, \ldots, \varphi_{m}\right\} \quad \text { and } \quad P_{m} v=\sum_{j=1}^{m}\left(v, \varphi_{j}\right) \varphi_{j}
$$

For $v \in D(A) \subset V$, we can write it

$$
v=P_{m} v+\left(I-P_{m}\right) v=P_{m} v+v_{2} .
$$

Set $\Theta=v_{1}(t)$ in $(2.1)$ to get

$$
\frac{1}{2} \frac{d}{d t}\left|v_{2}(t)\right|^{2}+\nu\left\|v_{2}(t)\right\|^{2} \leq j\left(v_{1}(t)\right)-j(v(t))-b\left(v(t), v(t), v_{2}(t)\right)+\left(\mathcal{L}(v(t)), v_{2}(t)\right) .
$$

From the continuity of the trace operator we have

$$
j\left(v_{1}(t)\right)-j(v(t)) \leq j\left(v_{2}(t)\right) \leq C+\frac{\nu}{4}\left\|v_{2}(t)\right\|^{2}
$$

Further, using the Ladyzhenskaya inequality (3.1) we easily arrive at

$$
\frac{d}{d t}\left|v_{2}(t)\right|^{2}+\nu\left\|v_{2}(t)\right\|^{2} \leq C_{2}\left(1+F(t)+\|v(t)\|^{2}\right) .
$$

and

$$
\frac{d}{d t}\left|v_{2}(t)\right|^{2}+\nu \lambda_{m+1}\left|v_{2}(t)\right|^{2} \leq C_{2}\left(1+F(t)+\|v(t)\|^{2}\right) .
$$

Now, let $\varepsilon>0$ ge given. Using Lemmas 4.1 and 4.2, and taking $m$ large enough, we obtain

$$
\left|\left(I-P_{m}\right) \mathbf{U}(s+t, t) v_{0}\right|^{2} \leq \varepsilon
$$

uniformly in $t$, for $v_{0} \in B(0, \rho)$ and all $s \geq s_{0}(\rho, \varepsilon)$ large enough. This ends the proof of the theorem.

\section{References}

[1] M. Boukrouche and R. El-Mir, On a non-isothermal, non-Newtonian lubrication problem with Tresca law. Existence and the behavior of weak solutions Nonlinear Anal. Real World Applications, in press.

[2] M. Boukrouche and G. Łukaszewicz, An upper bound on the attractor dimension of a $2 D$ turbulent shear flow in lubrication theory, Nonlinear Analysis 59 (2004), 1077-1089.

[3] M. Boukrouche and G. Łukaszewicz, An upper bound on the attractor dimension of a $2 D$ turbulent shear flow with a free boundary condition. in: Regularity and Other Aspects of the Navier-Stokes Equations, Banach Center Publ. 70, Polish Acad. Sci., Warsaw, 2005, $61-72$. 
[4] M. Boukrouche, G. Łukaszewicz and J. Real, On pullback attractors for a class of twodimensional turbulent shear flows, International Journal of Engineering Science 44 (2006), 830-844.

[5] T. Caraballo, G. Łukaszewicz and J. Real, Pullback attractors for non-autonomous 2DNavier-Stokes equations in some unbounded domains, C. R. Acad. Sci. Paris Sér. I 342 (2006), 263-268.

[6] T. Caraballo, G. Łukaszewicz and J. Real, Pullback attractors for asymptotically compact non-autonomous dynamical systems, Nonlinear Analysis 64 (2006), 484-498.

[7] G. Duvaut and J. L. Lions, Les inéquations en mécanique et en physique, Dunod, Paris, 1972.

[8] J. Haslinger, I. Hlavâcek and J. Nečas, Numerical methods for unilateral problems in solid mechanics, in: Handbook of Numerical Analysis, Vol. IV, edited by P. G. Ciarlet and J. L. Lions, 1996.

[9] K. Kuratowski, Sur les espaces complets, Fund. Math. 15 (1930), 301-309.

[10] H. Song and H. Wu, Pullback attractors of nonautonomous reaction-diffusion equations, J. Math. Anal. Appl. 325 (2007), 1200-1215.

[11] R. Temam, Infinite-Dimensional Dynamical Systems in Mechanics and Physics, 2nd ed., Springer-Verlag, New York, 1997. 
